\title{
Dynamics of Psychopathology Symptoms in Opiate-Dependent Patients During Therapy
}

\author{
Igumnov Sergey \\ Aleksandrovich \\ Serbsky Federal Medical Research \\ Centre of Psychiatry and Narcology, \\ Ministry of Health of the Russian \\ Federation, Moscow, Russia
}

\author{
Stan'ko Edward Pavlovich \\ Educational Institution, Grodno \\ State Medical University, Grodno, \\ Belarus
}

\author{
Nestsiarovich Anastasiya \\ Nikolayevna \\ Belarusian State Medical University, \\ Minsk, Belarus
}

The article provides the results of the clinical and psychological investigation of indicators related to psychopathological state of HIV-positive (HP) and HIV-negative (HN) patients with opiate dependence before and after the treatment at the stages of establishment and stabilization of remission. Objectives: to investigate the structure and dynamics of psychopathological symptoms in HIV-positive and HIV-negative patients with opiate dependence. Material and methods: Investigated sample included 820 patients. Severity of the major psychopathological symptoms was assessed in dynamics with the questionnaire SCL-90-R. The structure of psychopathological symptoms in patients with opiate dependence is presented. The changes of psychopathological indicators are revealed in the sample studied before and after the treatment course (1, 3, 6 and 12 months after the beginning of observation). Results: The findings demonstrate more severe psychopathology in HP patients versus HN ones. It was found that SCL-90-R scores in patients were higher than in healthy individuals, despite of their positive dynamics at discharge, indicating the failure of the short-term therapy course to normalize the mental state of patients with opiate dependence and the need to develop the algorithm for such patients, including the long-term, comprehensive programs of treatment and rehabilitation that are corrected according to the patient's needs. Conclusions: The course of the short-term treatment allows to achieve the clinical improvement at the stage of the remission establishment, but does not stabilize the mental state of the drug-dependent patients with HIV; that indicates the need to develop the long-term, comprehensive treatment and rehabilitation programs based on the patient's needs.

Keywords: psychopathology, dynamic assessment, opiate dependence, HIV

\section{Introduction}

Improving the effectiveness of treatment and the quality of remission in drug-dependent patients (DDP) is a complicated task for modern research in the area of addictive disorders. High prevalence of drug addiction, its common co-morbidity with mental disorders, the lack of willingness of addictive disorders services to meet the

Igumnov Sergey Aleksandrovich, M.D., professor, Serbsky Federal Medical Research Centre of Psychiatry and Narcology, Ministry of Health of the Russian Federation.

Stan'ko Edward Pavlovich, M.D., associate professor, Educational Institution, Grodno State Medical University.

Nestsiarovich Anastasiya Nikolayevna, M.D., Belarusian State Medical University. 
challenges of effective psychosocial rehabilitation of DDP make this problem topical (Ivanec \& Vinnikova, 2011; Ribakova, 2012). Providing DDP with mainly short-term assistance and incomplete coverage them with medical observation contribute to displacement of the comprehensive approach to drug dependence treatment. As a result, patients do not receive all the required amount of medical and rehabilitation care, which leads to disease progression and chronization (Krupicky, 2010; Magalif \& Magalif, 2003). Specialized care is limited to relief of withdrawal states or acute conditions related to psychoactive substances (PAS) consumption. As a result, $90 \%$ of DDP who underwent a single one year course of in-patient treatment renew the uncontrolled use of drugs, and more than $70 \%$ of them are admitted to hospital for treatment several times per year (Iliuk, 2012).

Substance abuse disorders, as well as other psychiatric disorders have a progressive nature. Their complete cure is an infeasible task. An inherent part of the disease is a relapse state that occurs among the trials to control the drug use. The long-term remission and recovery have to be achieved stepwise. The evidence of inadequacy of "complete refusal of drug use" criterion in assessment of the therapeutic result requires the development of methods for the dynamic evaluation of treatment effectiveness and remission quality with the use of multidimensional psychometric instruments. Symptoms of drug dependence constitute a wide range of disturbances related to "big" and "borderline" psychiatry with the evident unity of mental and somatic-neurological abnormalities. Mental disorders occur in 50\%-75\% of DDP and have an adverse effect on the quality of their remission. The most common psychopathological disturbances in the clinical structure of drug dependence are affective disturbances. Mood disorders range from mild melancholy to severe depressive states and contribute to the craving for PAS and to withdrawal state. PAS induced psycho-emotional disturbances interfere with the formation of a stable remission and lead to the disease relapse in $50 \%$ of cases (Iliuk, Krupicky, \& Shishkova, 2012). Intrinsic psychotropic effect of opiates influences the entire spectrum of mental and behavioral disorders that are complex and manifest with multifaceted symptomatology. Identification of certain psychopathological symptoms when choosing the treatment strategy may enhance the effectiveness of DDP treatment. It is of biggest interest the study of the structure and dynamics of psychopathological symptoms before and after the treatment, at the stage of follow-up observation to evaluate the treatment effectiveness and the remission quality in patients with opiate addiction.

The aim of the present study was to investigate the structure and dynamics of psychopathological symptoms in DDP before and after the treatment course, during the remission formation and stabilization.

\section{Material and Methods}

The main object of the study is represented by HIV-positive patients with opiate addiction, living on the territory of the Republic of Belarus. The subject of research is clinical, social and psychological characteristics of dependent patients with HIV infection (HIV-positive, HP) and without HIV infection (HIV-negative, HN) before and after the treatment at the stage of the remission formation and stabilization.

The present study is combined (cross-sectional and prospective) with regular monitoring (6-fold research) of studied psychopathological parameters before and after the treatment course during the follow-up period-1, 3, 6 and 12 months after the beginning of observation. The clinical study was performed in accordance with the GCP rules, according to the protocol with the use of the standardized registration card of patient (Good clinical practice, 2005).

The total of $376 \mathrm{HP}$ patients (I group) and $444 \mathrm{HN}$ patients (II group) were examined. The main group (I) included patients with the verified diagnosis of "opiate dependence" F11.2x and "HIV" B20, meeting the 
relevant ICD-10 criteria. The age of HP patients ranged from 13 to 53 years, the average age was 32.9 years $(S D=5.54)$; for males -33.7 years $(S D=5.59)$ and for females -30.8 years $(S D=4.86)$. Disease duration was 10 years and more. Patients who refused to participate in the survey were excluded from the observation group, as well as the patients in a state of withdrawal, with severe organic personality changes, acute psychotic disorder, exacerbation of mental illness or physical illness decompensation, mental retardation and schizophrenia. The control group included 65 healthy individuals (Minko, 2009). Verification of somatic pathology was conducted by the qualified experts from the staff of specialized medical institutions with modern diagnostic equipment.

The questionnaire Symptom Checklist-90-Revised (SCL-90-R) (Derogatis, Lipman, \& Covi, 1973; Derogatis, Rickels, \& Rock, 1976) in the adapted version of N. V. Tarabrina (2001) was used for multi-dimensional evaluation of psychopathology severity, after relief of the patient's withdrawal state. SCL-90-R is designed for patient's self-completion and does not require participation of a specialist. It consists of 90 items, each of which is estimated with five-point scale (from 0 to 4 ) where 0 is "Not at all" and 4 is "Very much". Answers related to these 90 points are calculated and interpreted under 9 major symptomatic scales: Somatization (SOM), Obsessive-Compulsive symptoms (O-C), Interpersonal Sensitivity (INT), Depression (DEP), Anxiety (ANX), Hostility (HOS), Phobic Anxiety (PHOB), Paranoid Ideation (PAR), Psychoticism (PSY).

Three general scales of the second order are also used: GSI — the global severity index; PTSD — positive symptom distress index; PST - positive symptom total. Results of the SCL-90-R can be interpreted at three levels: the overall severity of psychopathological symptoms, severity of individual scales and severity of individual symptoms.

Statistical analysis was performed with the use of the software package Statistica 10.0 (SN AXAR207F394425FA-Q). In order to describe the distribution of quantitative variables in the sample we used the mathematic expectation $(M)$ and standard deviation $(S D)$ format $M \pm S D$ or median (Me) and the interquartile range in the form of Me (LQ-UQ), where LQ is the lower quartile, UQ- the upper quartile. To compare the groups by the quantitative variables we used nonparametric methods: U Mann-Whitney test for independent samples and Wilcoxon test for related samples. Analyzed differences were considered as statistically significant at $p<0.05$.

\section{Results}

The prevalence of mental disorders among injecting drug users (IDUs) is 8 times higher than the average one in general population; $50 \%$ of those infected with HIV suffer from mental disorders (Sumarokova, 2009). HIV-positive subjects suffer from affective, cognitive and addictive disorders, dementia. Substance dependence and HIV mutually aggravate each other, which leads to a rapid progression of HIV infection. While increasing the disease severity, HIV infection increases the probability of psychotic disorders development in DDP. Combined HIV, drug addiction and mental disorders deteriorate the course of comorbid state, worsen the prognosis and increase the risk of spreading the infectious diseases and drug addiction. Personality disorders are found in $28.9 \%$ of DDP (Iliuk et al., 2012). Mental disorders have a negative impact on the therapeutic dynamics and the treatment efficacy and may determine the resistance in DDP (Bohan, Katkov, \& Rossinsky, 2005). It should be noted that the deterioration of craving for the drug often manifests itself with mental (affective) and behavior disturbances in DDP (Rohlina et al., 2002). 
Acute intoxication and withdrawal state occur in a more severe form in DDP with mental disorders than in DDP without ones. The presence of mental disorders in DDP makes it difficult to identify and control spontaneously arising primary craving for the drug in withdrawal state, provoking cognitive impairments in the form of thought disorganization and attention deficit (Bohan et al., 2005).

The studied sample was mostly represented by the patients with low levels of education and low rates of employment, problems at work and in the family, low moral qualities and criminal behavior. Patients indicated that drug dependence and health problems had affected their vitality and emotional state, the overall level of social functioning and the intensity of social contacts in particular. Evaluation of psychopathological symptoms in groups of patients revealed that scores in HP patients were higher before the treatment (see Table 1).

Table 1

Results of the Symptoms Severity Assessment in HP and HN Patients Prior to Treatment

\begin{tabular}{llllllllllll}
\hline SCL90R & \multicolumn{9}{c}{ I group $(n=376)$} \\
\cline { 2 - 5 } & Q25 & Med & Q75 & Means & $S D$ & Q25 & Med & Q75 & Means & $S D$ & \\
\hline SOM & 1.16 & 1.83 & 2.25 & 1.72 & 0.74 & 0.85 & 1.33 & 1.83 & 1.42 & 0.71 & 0.000000 \\
O-C & 0.80 & 1.40 & 1.90 & 1.34 & 0.66 & 0.60 & 1.10 & 1.50 & 1.13 & 0.66 & 0.000006 \\
INT & 0.66 & 1.11 & 1.66 & 1.30 & 0.72 & 0.55 & 1.00 & 1.55 & 1.07 & 0.71 & 0.000000 \\
DEP & 1.07 & 1.69 & 2.15 & 1.64 & 0.71 & 0.92 & 1.38 & 1.92 & 1.46 & 0.74 & 0.000578 \\
ANX & 0.90 & 1.60 & 2.10 & 1.55 & 0.79 & 0.70 & 1.10 & 1.80 & 1.26 & 0.75 & 0.000000 \\
HOS & 0.66 & 1.33 & 2.16 & 1.41 & 0.83 & 0.50 & 1.00 & 1.60 & 1.12 & 0.77 & 0.000001 \\
PHOB & 0.28 & 0.71 & 1.14 & 0.77 & 0.63 & 0.14 & 0.42 & 0.85 & 0.58 & 0.58 & 0.000004 \\
PAR & 1.00 & 1.50 & 2.00 & 1.53 & 0.75 & 0.66 & 1.00 & 1.67 & 1.22 & 0.76 & 0.000000 \\
PSY & 0.40 & 0.90 & 1.40 & 0.94 & 0.65 & 0.30 & 0.70 & 1.10 & 0.79 & 0.61 & 0.000435 \\
ADD & 1.14 & 1.71 & 2.14 & 1.65 & 0.75 & 1.00 & 1.57 & 2.14 & 1.59 & 0.78 & 0.180215 \\
GSI & 0.91 & 1.47 & 1.88 & 1.40 & 0.61 & 0.77 & 1.15 & 1.60 & 1.19 & 0.60 & 0.000000 \\
\hline
\end{tabular}

Notes. SOM: Somatization, O-C: Obsessive-Compulsive, INT: Interpersonal Sensitivity, DEP: Depression, ANX: Anxiety, HOS: Hostility, PHOB: Phobic Anxiety, PAR: Paranoid Ideation, PSY: Psychoticism, ADD: Additional Scale, GSI: Global Severity Index.

As can be seen from Table 1, the comparison of HP and HN patients before the treatment with Mann-Whitney test has revealed significant differences: All scores of SCL-90-R scale were significantly lower in HN patients, compared to the HP ones. Noteworthy is the fact that statistically significant differences at the level of $p<0.0005$ were related to all of the questionnaire items (except for the values of the additional scale). The highest scores of SCL-90-R were observed in HP patients prior to treatment on the scales of somatization, depression, anxiety, paranoid ideation and hostility, the lowest scores — on the scales of obsessive-compulsive symptoms, interpersonal sensitivity, psychoticism and phobic anxiety. The Global severity index in HP patients before the treatment was $15 \%$ higher than the one in $\mathrm{HN}$ patients (respectively, $1.40(\mathrm{SE}=0.61)$ and $1.19(\mathrm{SE}=$ $0.60)$.

Despite of the treatment course, the highest scores that HP patients displayed at discharge were found on the scales of "paranoid ideation", "depression", "somatization" and "anxiety", the lower scores were found on the scales "obsessive-compulsive symptoms", "hostility", "psychoticism" and "phobic anxiety" (see Table 2).

As can be seen from the Tables 1 and 2, the most prominent clinical differences in HP patients prior to treatment and after it were found on the scale "somatization" (1.7 times) and this score was within the range of Me 0.95 (0.50-1.41) at discharge. The levels of obsessive-compulsive symptoms, hostility, depression and 
anxiety have reduced 1.5 times at discharge in HP patients; the interpersonal sensitivity, phobic anxiety and psychoticism have reduced 1.4 times and paranoid ideation has reduced 1.3 times. The Global severity index has decreased 1.5 times, but its inter-quartile range has remained within Me 0.88 (0.46-1.41) in HP patients at discharge.

The data obtained have shown that, despite the significant reduction in the severity of psychopathological symptoms of all SCL-90-R scales, their average values in HP patients were still higher than those ones in HN patients and healthy individuals after the treatment course.

Table 2

Results of the Symptoms Severity Assessment in HP and HN Patients After the Treatment (at Discharge)

\begin{tabular}{llllllllllll}
\hline \multirow{2}{*}{$\begin{array}{l}\text { SCL90R } \\
\text { subscales }\end{array}$} & \multicolumn{9}{c}{ I group $(n=376)$} & \multicolumn{7}{c}{ II group $(n=444)$} \\
\hline NOM & Q25 & Med & Q75 & Means & $S D$ & Q25 & Med & Q75 & Means & $S D$ \\
O-C & 0.50 & 0.95 & 1.41 & 1.02 & 0.64 & 0.41 & 0.75 & 1.16 & 0.80 & 0.53 & 0.000038 \\
INT & 0.40 & 0.83 & 1.40 & 0.88 & 0.57 & 0.40 & 0.70 & 1.10 & 0.80 & 0.50 & 0.067729 \\
DEP & 0.44 & 0.88 & 1.33 & 0.93 & 0.64 & 0.44 & 0.67 & 1.00 & 0.76 & 0.52 & 0.001249 \\
ANX & 0.53 & 1.00 & 1.53 & 1.11 & 0.65 & 0.53 & 0.84 & 1.31 & 0.98 & 0.58 & 0.021569 \\
HOS & 0.50 & 0.90 & 1.50 & 1.03 & 0.66 & 0.40 & 0.70 & 1.10 & 0.80 & 0.50 & 0.000047 \\
PHOB & 0.33 & 0.83 & 1.50 & 0.94 & 0.72 & 0.33 & 0.66 & 1.00 & 0.69 & 0.49 & 0.000200 \\
PAR & 0.14 & 0.57 & 0.85 & 0.54 & 0.47 & 0.14 & 0.28 & 0.57 & 0.37 & 0.36 & 0.000033 \\
PSY & 0.50 & 1.16 & 1.66 & 1.14 & 0.76 & 0.50 & 0.83 & 1.33 & 0.91 & 0.62 & 0.000147 \\
ADD & 0.20 & 0.60 & 1.00 & 0.68 & 0.52 & 0.20 & 0.50 & 0.80 & 0.59 & 0.46 & 0.028310 \\
GSI & 0.71 & 1.14 & 1.42 & 1.08 & 0.56 & 0.57 & 1.00 & 1.42 & 1.06 & 0.62 & 0.176426 \\
\hline & 0.46 & 0.88 & 1.41 & 0.94 & 0.54 & 0.50 & 0.75 & 1.04 & 0.79 & 0.42 & 0.000610 \\
\hline
\end{tabular}

Notes. SOM: Somatization, O-C: Obsessive-Compulsive, INT: Interpersonal Sensitivity, DEP: Depression, ANX: Anxiety, HOS: Hostility, PHOB: Phobic Anxiety, PAR: Paranoid Ideation, PSY: Psychoticism, ADD: Additional Scale, GSI: Global Severity Index.

The pre-treatment value of the global severity index was 6.4 times higher in HP patients than in controls and 5.4 higher in $\mathrm{HN}$ patients than in controls. After treatment, the same values were respectively 4.3 times higher in HP patients and 3.6 times higher in HN patients compared to controls.

One month after the beginning of the observation period, the severity of psychopathology in HP patients has grown with the increase of GSI values to Me 0.88 (0.46-1.41), in contrast to HN patients in whom this parameter has gradually reduced. Later, after 3, 6 and 12 months of observation, the curve of the Global severity index in HP patients had been maintaining the same level, with the value almost 5 times higher than in controls. After 12 months of the follow-up period the difference in the global severity index between HP and $\mathrm{HN}$ patients has reached 1.5 times.

The data obtained indicate not only the presence of remaining psychopathology, but also its severity which reflects the prominence of the distress experienced by patients of the studied groups after the treatment course during the formation and stabilization of remission. In such circumstances, the short-term course of therapy improves but does not stabilizes the mental state of DDP, especially of those with HIV, which increases the risk of drug dependence relapse and promotes the chronization of the existing mental disorders.

Analysis of SCL-90-R scales in patients of both groups before and after the treatment course after 1, 3, 6 and 12 months from the beginning of the observation period has shown that the highest values with the maximum severity of psychopathological symptoms on every scale were typical for HP patients. 
The highest SCL-90-R scores in both patient groups were those for somatization, depression, anxiety, paranoid ideation and hostility. However, the values of the studied parameters differed among the comparison groups. Somatization score differed 1.2 times between HP and HN patients, 5.2 times between HP patients and healthy people, 4.3 times between HN patients and controls. The level of depression was 8.6 times higher in HP patients than in controls and 7.7 times higher in HN patients than in healthy individuals. The level of anxiety was 10.4 times higher in HP patients than in controls and 8.4 times higher in HN patients than in controls. Paranoid ideation differed 1.3 times between HP and HN patients and was 6.7 times more prominent in HP patients compared to controls and 5.3 times more prominent in $\mathrm{HN}$ patients than in controls.

The severity of psychopathological symptoms in patients of both groups has declined after the treatment course at discharge. The severity of symptoms has reduced after the treatment course; nevertheless the average values of the studied parameters in HP and HN patients were still higher than those ones in the control group. The greatest psychopathology reduction in HP patients after the treatment was observed in somatization (1.7 times), obsessive-compulsive symptoms, anxiety, hostility and depression (1.5 times), to a lesser extent - paranoid ideation and psychoticism (1.3), phobic anxiety and interpersonal sensitivity (1.4). Reduction of symptoms in HN patients was observed on scales of somatization (1.8 times), hostility and anxiety (1.6 times), phobic anxiety and depression (1.5 times), to a lesser extent—paranoid ideation and psychoticism (1.3), interpersonal sensitivity (1.4).

The highest SCL-90-R scores were observed on depression, anxiety, and paranoid ideation scales. Despite of the somatization level had reduced 1.7 times in HP patients and 1.8 times in HN patients, its values were still 2.4 times and 2 times higher (respectively) than in control group. The level of depression has become 2 times less in patients from both groups at the moment of discharge, but its magnitude was still 5.8 times higher in HP patients and 5.2 times higher in $\mathrm{HN}$ patients compared to the control group. Anxiety became 1.5 times less severe in both groups of patients at discharge, but its value was still 7 times higher in HP patients and 5 times higher in HN patients compared to the controls. It should be noted that the level of paranoid ideation has changed slightly in HP and HN patients after the treatment course. With the total decrease of 1.3 times at discharge its value was still 5 times and 4 times (respectively) higher compared to the healthy individuals. The greatest differences between patients and controls were found on the scales of psychoticism and phobic anxiety. The level of psychoticism in HP patients was 14 times higher and in HN patients 12 times higher compared to controls at discharge, the differences for the phobic anxiety were, respectively, 9 times in HP patients and 6 times in $\mathrm{HN}$ patients.

The dynamic assessment of SCL-90-R scores 1, 3, 6 and 12 months after the beginning of the observation period indicates no significant changes of severity of psychopathological symptoms in HP and HN patients. However, the symptoms severity was different compared to its level before the treatment course.

The reduction in symptoms severity (except for somatization and phobic anxiety) is observed in the group of $\mathrm{HN}$ patients and its increase is observed in the group of HP patients after 12 months of the follow-up period. HP patients demonstrated exacerbation of psychopathological symptoms within the first month of the observation period and its dynamics had remained at the same level after 3, 6 and 12 months. The period of formation and stabilization of remission in the group of HP patients is associated with the increase of all SCL-90-R scores: paranoid ideation, somatization, anxiety, depression and hostility, to a lesser extent-interpersonal sensitivity, obsessive-compulsive symptoms, psychoticism and phobic anxiety. 
The comparative assessment of SCL-90-R scores before the treatment and 1, 3, 6 and 12 months after the treatment has showed the decrease in all scales values in the studied groups of patients. The differences at admission and 12 months after the treatment in the group of HP patients were 1.4 times for somatization scale, 1.5 times for depression scale and 1.3 times for the Global severity index; the differences in the group of HN patients were 1.8 times for depression and anxiety, 1.7 times for somatization, 1.6 times for hostility and psychoticism, 1.7 times for Global severity index.

The analysis of SCL-90-R values relationship at the moment of discharge and 12 months after the treatment has showed the dynamic differences in symptoms severity in HP and HN patients. Somatization differed 1.2 times between HP and HN patients at discharge, and 1.4 times after 12 months (1.2/1.4); anxiety ratio was $1.2 / 1.7$; depression ratio was $1.1 / 1.4$; paranoid ideation and interpersonal sensitivity ratios were $1.2 / 1.6$; hostility ratio was $1.2 / 1.6$; phobic anxiety ratio-1.3/1.7; psychoticism ratio-1.2/1.7; obsessive-compulsive symptoms ratio-1.2/1.3, Global severity index ratio was 1.2/1.5.

\section{Discussion}

Active drug consumption by addicted patients is associated with the development of mental disorders of varying severity. Failure to comply with the basic principles of therapy, to take into account the peculiarities of the substance abuse, the lack of effective methods of treatment and rehabilitation and its monitoring results in a low efficiency of the existing system of treatment and medical and social rehabilitation of drug-dependent patients (Rohlina et al., 2002; Stan'ko, Igumnov, \& Nestiarovich, 2015; Igumnov \& Stan'ko, 2015).

Dynamics of the SCL-90-R scores is quite informative which is important to take into account during the treatment process when assessing the risk of adverse events. The comparative assessment of SCL-90-R parameters, revealing the ratio of different problems, makes it possible to differentiate patients into groups according to the severity of psychiatric symptoms and disease on the whole, allows to select the priority strategies of therapy (medication, psychotherapy, psychosocial interventions) and to optimize the management of patients with opiate dependence that affects the course and prognosis of the disease.

The presence of co-morbid states (HIV, mental illness) has limited the efficacy of therapy. Despite the reduction of the SCL-90-R scores after the treatment course, their values in HP patients are still significantly higher than in HN patients and healthy individuals, indicating the failure of the short-term treatment course to normalize the mental state and the need for development of the algorithm of HP patients management, which includes the long-term, comprehensive treatment and rehabilitation programs that are corrected according to the patient's needs.

Early detection and treatment of co-morbid states (mental and somatic disorders) may reduce the likelihood of relapse and increase the effectiveness of the treatment programs. It is reasonable to use the multi-dimensional valid tools that comprehensively evaluate the entire spectrum of health and social consequences of the drug use to monitor the status of DDP.

Thus, the results of the study allow us to formulate the following conclusions.

Psychopathological symptoms, reflecting the level of the distress experienced, are typical for the patients with opiate dependence with and without HIV.

HIV-positive patients have statistically significant differences in symptoms severity $(p<0.0005)$ compared to HIV-negative patients and healthy individuals, indicating the pervasive pattern of the negative effect of opiates and HIV on mental state. 
The severity of psychopathology in HP patients is changeable: It is reduced after the treatment course and tends to exacerbate during the formation and stabilization of remission that increases the risk of relapse and indicates the low quality of remission as the result of ineffectiveness of the short-term therapy.

The course of the short-term treatment allows to achieve the clinical improvement at the stage of the remission establishment, but does not stabilize the mental state of the drug-dependent patients with HIV; that indicates the need to develop the long-term, comprehensive treatment and rehabilitation programs based on the patient's needs.

\section{References}

Bohan, N. A., Katkov, A. L., \& Rossinsky, J. A. (2005). Early prophylactic and rehabilitation of patients with opioid dependence. Pavlodar. (In Russian)

Derogatis, L. R., Lipman, R. S., \& Covi, L. (1973). SCL-90: An outpatient psychiatric rating scale (Preliminary report). Psychopharmacology Bulletin, 9(1), 13-27.

Derogatis, L. R., Rickels, K., \& Rock, A. (1976). The SCL-90 and the MMPI: A step in the validation of a new self-report scale. Brit. J. Psychiat, 128, 280-289.

Good clinical practice. (2005). National Standard of the Russian Federation: State Standard 52379-2005. Moscow: Federal Agency of Technical Regulation and Metrology: National Standard of the Russian Federation (Electronic source). Retrieved from http://www.medtran.ru/rus/trials/gost/52379-2005.htm (In Russian)

Iliuk, R. D. (2012). Dynamic assessment of clinical and social characteristic of injecting drug users in rehabilitation treatment: Manual for doctors. Saint Petersburg. (In Russian)

Iliuk, R. D., Krupicky, E. M., \& Shishkova, A. M. (2012). The program of the complex therapy of comorbid psychopathological and behavioral disorders in HIV-positive drug-dependent patients: Methodological recommendations. Saint Petersburg Research Psychoneurological Institute of V.M. Bechterev. Saint Petersburg. (In Russian)

Ivanec, N. N., \& Vinnikova, M. A. (2011). Alcoholism: The manual for doctors. Moscow: Medical information agency. (In Russian)

Krupicky, E. M. (2010). The short-term intensive psychotherapeutical intervention in narcology according to the evidence based approach. Herald of Neurology, 42(3), 25-27. (In Russian)

Magalif, A. J., \& Magalif, A. A. (2003). Existential depression and alcoholism. Affective and schizoaffective disorders: Materials of the conference. Moscow. (In Russian)

Minko, O. O. (2009). Informativeness and diagnostic value of the results of psychodiagnostic assessment as the markers of opioid dependence. Ukrainian Herald of Psychoneurology, 2(59), 35-39. (In Ukrainian)

Ribakova, K. V. et al. (2012). The assessment of the remission quality in patients with alcohol dependence based on the clinical and social-psychological criteria of re-adaptation to sobriety. Review of Psychiatry and Medical Psychology, 4, 63-72. (In Russian)

Rohlina, M. L. et al. (2002). Principles of pharmacotherapy of opioid narcomany. Narcology, 11, 28-30. (In Russian)

Stan'ko, E. P., Igumnov, S. A., Nestiarovich, A. (2015). Dynamics of the life quality parameters in HIV-positive patients with opiate dependence. Psychology Research, 5(3), 205-212.

Sumarokova, M. A. (2009). Epidemiological and psychopathological aspects of HIV problem in injecting drug users. Narcology, 8(10), 75-80. (In Russian)

Tababrina, N. V. (2001). Practical recommendations on psychology of posttraumatic stress. Saint Petersburg: "Piter". (In Russian)

Igumnov, S. A., \& Stan'ko, E.P. (2015). Medical and psychosocial aspects of injecting drug use by patients with HIV infection. Applied Psychology and Psychotherapy, 1, 57-58. 